\title{
Crystal nucleation of colloidal hard dumbbells
}

\author{
Ran $\mathrm{Ni}^{\mathrm{a})}$ and Marjolein Dijkstrab) \\ Soft Condensed Matter, Debye Institute for NanoMaterials Science, Utrecht University, Princetonplein 5 , \\ $3584 C C$ Utrecht, The Netherlands
}

(Received 27 October 2010; accepted 29 November 2010; published online 18 January 2011)

\begin{abstract}
Using computer simulations, we investigate the homogeneous crystal nucleation in suspensions of colloidal hard dumbbells. The free energy barriers are determined by Monte Carlo simulations using the umbrella sampling technique. We calculate the nucleation rates for the plastic crystal and the aperiodic crystal phase using the kinetic prefactor as determined from event driven molecular dynamics simulations. We find good agreement with the nucleation rates determined from spontaneous nucleation events observed in event driven molecular dynamics simulations within error bars of one order of magnitude. We study the effect of aspect ratio of the dumbbells on the nucleation of plastic and aperiodic crystal phases, and we also determine the structure of the critical nuclei. Moreover, we find that the nucleation of the aligned close-packed crystal structure is strongly suppressed by a high free energy barrier at low supersaturations and slow dynamics at high supersaturations. (C) 2011 American Institute of Physics. [doi:10.1063/1.3528222]
\end{abstract}

\section{INTRODUCTION}

Recent breakthroughs in particle synthesis produced a spectacular variety of anisotropic building blocks. ${ }^{1}$ Colloidal particles with the shape of a dumbbell are one of the simplest anisotropic building blocks. Their unique morphologies lead to novel self-organized structures. For instance, it was found that magnetic colloidal dumbbells can form chainlike clusters with tunable chirality, ${ }^{2}$ while novel crystal structures have been predicted recently for asymmetric dumbbell particles consisting of a tangent large and small hard sphere, which are atomic analogs of $\mathrm{NaCl}, \mathrm{CsCl}, \gamma \mathrm{CuTi}, \mathrm{CrB}$, and $\alpha \mathrm{IrV}$, when we regard the two individual spheres of each dumbbell independently. ${ }^{3}$ Moreover, colloidal dumbbells also gain increasing scientific attention in recent years due to its potential use in photonic applications. It has been shown that dumbbells on a face-centered-cubic lattice, where the spheres of the dumbbells form a diamond structure, exhibit a complete band gap, ${ }^{4,5}$ while it is impossible to obtain a complete band gap in systems consisting of spherical particles. A very recent calculation showed that for midrange aspect ratios, both asymmetric and symmetric dumbbells have 2-3 large band gaps in the inverted lattice. ${ }^{6}$ Although these structures are not thermodynamically stable for hard dumbbells, ${ }^{7-13}$ it does show the promising potential of anisotropic particles in photonic applications.

New routes of synthesizing colloidal dumbbells make it easy to control the aspect ratio. ${ }^{14}$ In addition, by adding salt to the solvent, the interactions between dumbbells can be tuned from long-ranged repulsive to hard interactions. Although hard dumbbells were originally modeled for simple nonspherical diatomic molecules, such as nitrogen, they are also a natural model system for studying the self-assembly of colloidal dumbbells. ${ }^{15-18}$ The phase behavior of hard dumbbells has

a)Electronic mail: r.ni@uu.nl.

b) Electronic mail: m.dijkstra1 @uu.nl. been extensively studied by density functional theory ${ }^{7,8}$ and computer simulations. ${ }^{9-13}$ The bulk phase diagram of hard dumbbells displays three types of stable crystal structures. ${ }^{7-13}$ For small aspect ratio, the dumbbells form a plastic crystal phase at low densities. The freezing into a cubic plastic crystal phase in which the dumbbells are positioned on a facecentered-cubic lattice, but are free to rotate, has been determined using Monte Carlo (MC) simulations. ${ }^{9}$ These results have been refined by Vega, Paras, and Monson, who showed that at higher densities the cubic plastic crystal phase transforms into an orientationally ordered close-packed (CP) crystal structure, which they labeled CP1. Additionally, these authors showed that the fluid-cubic plastic crystal coexistence region terminates at $L / \sigma \simeq 0.38$, where $L$ is the distance between the centers of spheres and $\sigma$ is the diameter of the dumbbells. For longer dumbbells a fluid-CP1 coexistence region was found, whereas the relative stability of the closepacked crystal structures $\mathrm{CP} 1, \mathrm{CP} 2$, and CP3, which only differ in the way the hexagonally packed dumbbell layers are stacked, remained undetermined as the free energies are very similar. ${ }^{10-12}$ Moreover, these authors showed by making an estimate for the degeneracy contribution to the free energy that dumbbells with $L / \sigma=1$ may form an aperiodic crystal phase. ${ }^{10}$ The stability of such an aperiodic crystal structure in which both the orientations and positions of the particles are disordered, while the spheres of each dumbbell are located on the lattice positions of a random-hexagonal-close-packed (rhcp) lattice has been verified recently for $L / \sigma>0.88 .{ }^{13} \mathrm{In}$ addition, it has been shown that the plastic crystal phase with the hexagonal-close-packed structure is more stable than the cubic plastic crystal for a large part of the stable plastic crystal region. ${ }^{13}$ Although, the bulk phase diagram is well studied, the kinetic pathways of the fluid-solid phase transitions are still unknown, and only a few studies have been devoted to the crystal nucleation of anisotropic particles. ${ }^{19,20}$ In the present work, we investigate the nucleation of the plastic crystal phase of hard dumbbells using computer simulations and study 
the effect of aspect ratio of the dumbbells on the resulting nucleation rates and the structure and size of the critical nuclei. Moreover, for longer dumbbells we investigate crystal nucleation of the aperiodic crystal phase. First, we calculate the free energy barriers for nucleation using Monte Carlo simulations with the umbrella sampling technique, which are then combined with event driven molecular dynamics (EDMD) simulations to determine the kinetic prefactor and the nucleation rates. Additionally, we determine the nucleation rates from spontaneous nucleation events observed in EDMD simulations. We compare the nucleation rates and critical nuclei obtained from the umbrella sampling MC simulations with those from EDMD simulations.

The remainder of this paper is organized as follows. In Sec. II, we describe the methodology including the model and simulation methods used. We present the results and discussions on the nucleation of three types of crystal phases in suspensions of hard dumbbells in Sec. III. We end with some discussions and conclude in Sec. IV.

\section{METHODOLOGY}

We consider a system of hard dumbbells consisting of two overlapping hard spheres with diameter $\sigma$ with the centers separated by a distance $L$. We define the aspect ratio as $L^{*} \equiv L / \sigma$, such that the model reduces to hard spheres for $L^{*}=0$ and to tangent spheres for $L^{*}=1$. We study crystal nucleation of hard dumbbells for $0 \leq L^{*} \leq 1$. We focus on the nucleation of the plastic crystal phase $\left(0 \leq L^{*}<0.4\right)$ and the aperiodic crystal phase $\left(0.88<L^{*} \leq 1\right){ }^{8,10-13}$

\section{A. Order parameter}

In order to study the nucleation of the crystal phase, we require a cluster criterion that identifies the crystalline clusters in a metastable fluid. In this work, we employ the order parameter based on the local bond order parameter analysis of Steinhardt et al. ${ }^{21}$ We define for every particle $i$, a $2 l+1$ dimensional complex vector $\mathbf{q}_{l}(i)$ given by

$$
q_{l m}(i)=\frac{1}{N_{b}(i)} \sum_{j=1}^{N_{b}(i)} \Upsilon_{l m}\left(\hat{\mathbf{r}}_{i j}\right),
$$

where $N_{b}(i)$ is the total number of neighboring particles of particle $i$, and $\Upsilon_{l m}\left(\hat{\mathbf{r}}_{i j}\right)$ is the spherical harmonics for the normalized direction vector $\hat{\mathbf{r}}_{i j}$ between particles $i$ and $j, l$ is a free integer parameter, and $m$ is an integer that runs from $m=-l$ to $m=+l$. Neighbors of particle $i$ are defined as those particles which lie within a given cut-off radius $r_{c}$ from particle $i$. In order to determine the correlation between the local environments of particles $i$ and $j$, we define the rotationally invariant function $d_{l}(i, j)$,

$$
d_{l}(i, j)=\sum_{m=-l}^{l} \tilde{q}_{l m}(i) \cdot \tilde{q}_{l m}^{*}(j),
$$

where $\tilde{q}_{l m}(i)=q_{l m}(i) /\left.\sqrt{\sum_{m=-l}^{l} \mid q_{l m}(i)}\right|^{2}$ and the asterisk is the complex conjugate. ${ }^{22}$ If $d_{l}(i, j)>d_{c}$, the bond between particles (sphere) $i$ and $j$ is regarded to be solidlike or connected, where $d_{c}$ is the dot-product cutoff. We identify a particle (sphere) as solidlike when it has at least $\xi_{c}$ solidlike bonds. We have chosen the symmetry index $l=6$ as the particles (spheres) display hexagonal order in the plastic crystal and the aperiodic crystal phase. We have chosen $r_{c}=1.3 \sigma, d_{c}$ $=0.7$, and $\xi_{c}=6$ in our simulations. It has been shown recently that the choice of order parameter $\left(r_{c}, d_{c}\right.$, and $\left.\xi_{c}\right)$ does not affect the resulting nucleation rate if it is not too restrictive. ${ }^{20,23}$

To analyze the structure of the critical nuclei, we use the averaged local bond order parameter $\bar{q}_{l}$ and $\bar{w}_{l}$ proposed by Lechner and Dellago, ${ }^{24}$ which allows us to identify each particle as fcc-like or hcp-like, provided the number of neighboring particles $N_{b}(i) \geq 10$ :

$$
\begin{aligned}
& \bar{q}_{l}(i)=\sqrt{\frac{4 \pi}{2 l+1} \sum_{m=-l}^{l}\left|\bar{q}_{l m}(i)\right|^{2}} \\
& \bar{w}_{l}(i)=\frac{\sum_{m_{1}+m_{2}+m_{3}=0}\left(\begin{array}{ccc}
l & l & l \\
m_{1} & m_{2} & m_{3}
\end{array}\right) \bar{q}_{l m_{1}}(i) \bar{q}_{l m_{2}}(i) \bar{q}_{l m_{3}}(i)}{\left(\sum_{m=-l}^{l}\left|\bar{q}_{l m}(i)\right|^{2}\right)^{3 / 2}}
\end{aligned}
$$

where

$$
\bar{q}_{l m}(i)=\frac{1}{N_{b}(i)+1} \sum_{k=0}^{N_{b}(i)} q_{l m}(i) .
$$

The sum from $k=0$ to $N_{b}(i)$ runs over all neighbors of particle (sphere) $i$ plus the particle (sphere) $i$ itself. While $q_{l m}(i)$ takes into account the structure of the first shell around particle $i$, the averaged $\bar{q}_{l m}(i)$ contains also the information of the structure of the second shell, which increases the accuracy of the crystal structure determination. In order to distinguish fcc-like and hcp-like particles, we employ $\bar{q}_{4}$ and $\bar{w}_{4}$, as the order parameter distributions of pure fcc and hcp phases of Lennard-Jones and Gaussian core systems are well separated in the $\bar{q}_{4}-\bar{w}_{4}$ plane. $^{24}$

\section{B. Umbrella sampling}

The Gibbs free energy $\Delta G(n)$ for the formation of a crystalline cluster of size $n$ is given by $\Delta G(n) / k_{B} T$ $=$ const $-\ln [P(n)]$, where $P(n)$ is the probability distribution function of finding a cluster of size $n, k_{B}$ is Boltzmann's constant, and $T$ the temperature. As nucleation is a rare event and the probability to find a spontaneous nucleation event is very small in a brute force simulation within a reasonable time, one has to resort to specialized simulation techniques such as forward flux sampling, umbrella sampling, or transition path sampling. Here, we employ the method developed by Auer and Frenkel ${ }^{25}$ to calculate the free energy of the largest cluster. In this method, the sampling is biased toward configurations that contain clusters with a certain size. To this end, we introduce a biasing potential $\omega\left(\mathbf{r}^{N}\right)$, which is a harmonic function of the cluster size $n$ :

$$
\beta \omega\left(\mathbf{r}^{N}\right)=\frac{1}{2} k\left[n\left(\mathbf{r}^{N}\right)-n_{0}\right]^{2},
$$


where $n\left(\mathbf{r}^{N}\right)$ is the size of largest cluster and $n_{0}$ is the center of the umbrella sampling window whose width depends on $k$. In this work we set $k=0.2$. By increasing the value of $n_{0}$, we increase the size of the largest crystalline cluster in our system, which enables us to cross the nucleation barrier. If we define the average number of crystalline clusters with $n$ particles by $\left\langle N_{n}\right\rangle$, one can calculate the probability distribution $P(n)=\left\langle N_{n}\right\rangle / N$ from which we can determine the Gibbs free energy $\Delta G(n)$.

\section{Event driven molecular dynamics simulations}

Since the potential between particles in systems of hard dumbbells is discontinuous, the pair interactions only change when particles collide. The particles perform elastic collisions when they encounter each other. We numerically identify and handle these collisions by using an EDMD simulation. ${ }^{26,27}$

Using MD simulations to determine the nucleation rate is straightforward. Starting with an equilibrated fluid configuration, the MD simulation is used to evolve the system until the largest cluster in the system exceeds the critical nucleus size. Then the nucleation rate is given by

$$
I=\frac{1}{\langle t\rangle V},
$$

where $\langle t\rangle$ is the averaged waiting time of forming a critical nucleus in a system of volume $V$.

\section{RESULTS AND DISCUSSIONS}

In this section, we present the results on the nucleation of the plastic crystal, the aperiodic crystal and the CP1 crystal phase in suspensions of hard dumbbells.

\section{A. Nucleation of the plastic crystal phase}

We first investigate the nucleation of the plastic crystal phase of hard dumbbells. Monte Carlo simulations with the umbrella sampling technique are performed on hard dumbbell fluids with $L^{*}=0,0.15$ and 0.3 at supersaturation $\beta|\Delta \mu|$ $=0.34$ and with $L^{*}=0,0.15$, and 0.2 for $\beta|\Delta \mu|=0.54$ with $\beta=1 / k_{B} T$. We have chosen a shorter aspect ratio for the highest supersaturation as the plastic crystal phase for dumbbells with $L^{*}=0.3$ becomes metastable with respect to the aligned $\mathrm{CP} 1$ phase for $P^{*}=P \sigma^{3} / k_{B} T>30$, i.e., $\beta|\Delta \mu|>0.47$. The Gibbs free energy $\beta \Delta G(n)$ as a function of cluster size $n$ is shown in Fig. 1. We clearly observe that at low supersaturation, i.e., $\beta|\Delta \mu|=0.34$, the heights of the free energy barriers increase slightly $(\sim 8 \%)$ with aspect ratio. More specifically, $\beta \Delta G^{*}=42.9 \pm 0.3,44.5$ \pm 1.1 , and $45.2 \pm 2$ for $L^{*}=0,0.15$ and 0.3 , respectively. According to classical nucleation theory (CNT), the nucleation barrier for a spherical nucleus with radius $R$ is given by $\Delta G(R)=4 \pi \gamma R^{2}-4 \pi|\Delta \mu| \rho_{s} R^{3} / 3$ with $\gamma$ the interfacial tension, $|\Delta \mu|$ the chemical potential difference between the solid and fluid phase, and $\rho_{s}$ the bulk density of the solid phase. CNT predicts a nucleation barrier height $\Delta G^{*}$ $=(16 \pi / 3) \gamma^{3} /\left(\rho_{s}|\Delta \mu|\right)^{2}$ and a critical radius $R^{*}$ $=2 \gamma / \rho_{s}|\Delta \mu|$. The small increase in barrier height with

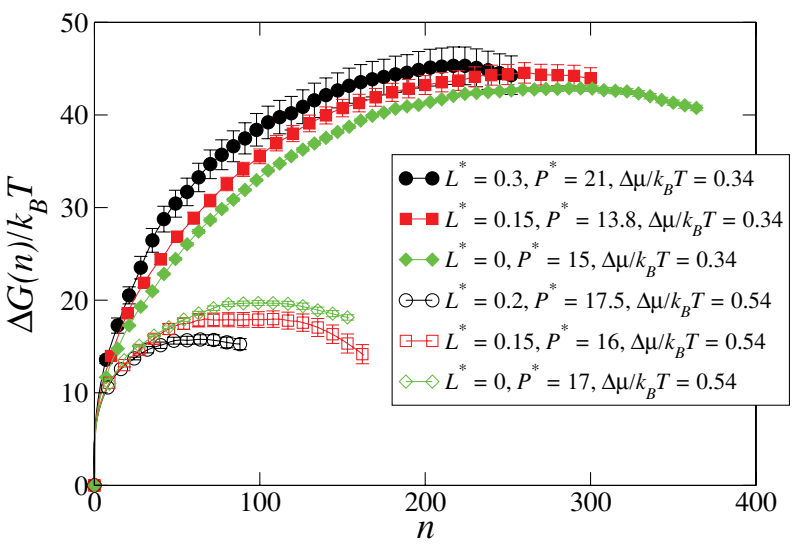

FIG. 1. Gibbs free energy $\Delta G(n) / k_{B} T$ as a function of cluster size $n$ for the nucleation of the plastic crystal phase of hard dumbbells with various aspect ratios $L^{*}=L / \sigma$ as displayed and supersaturation $\beta|\Delta \mu|=0.34$ (filled symbols) and 0.54 (open symbols).

aspect ratio can be explained by the small increase in the crystal-melt interfacial tensions that have been determined recently for the crystal planes (100), (110), and (111) using nonequilibrium work measurements with a cleaving procedure in MC simulations. ${ }^{28}$ For a spherical cluster, the surface tension is expected to be an average over the crystal planes, i.e., $\beta \gamma d^{2}=0.58,0.57$, and 0.60 , for $L^{*}=0,0.15$ and 0.3 , respectively, where $d^{3}=\sigma^{3}\left(1+3 / 2 L^{*}-1 / 5 L^{* 3}\right)$. Another work by Davidchack et al. found a slightly lower value for the averaged interfacial tension of hard spheres, i.e., $\beta \gamma d^{2}$ $=0.559 .{ }^{29}$ Using $\beta \Delta G^{*}=42.9$ and the more precise value for the surface tension $\beta \gamma d^{2}=0.559$, and the values for $\beta \gamma d^{2}$ and the bulk density $\rho_{s}$ for varying $L^{*}$ presented in Table I, CNT predicts a slightly larger increase in barrier height upon increasing $L^{*}$, i.e., $\beta \Delta G^{*}=45.6$ and 50.49, for $L^{*}=0.15$ and 0.3 , respectively. However, when the supersaturation is increased to $\beta|\Delta \mu|=0.54$, we find a decrease in barrier height upon increasing the aspect ratio as shown in Fig. 1 and Table I, which cannot be explained by CNT. Apparently, the pressure dependence of the surface tension is different for dumbbells with various aspect ratios.

The nucleation barriers obtained from umbrella sampling MC simulations can also be used to determine the nucleation rates as given by ${ }^{25}$

$$
I=\kappa \exp \left(-\beta \Delta G^{*}\right)
$$

where $\kappa$ is the kinetic prefactor given by $\kappa$ $=\rho_{l} f_{n^{*}} \sqrt{\left|\Delta G^{\prime \prime}\left(n^{*}\right)\right| / 2 \pi k_{B} T}, \quad \rho_{l}$ is the number density of particles in the fluid phase, $f_{n^{*}}$ the rate at which particles are attached to the critical nucleus, $\Delta G^{\prime \prime}\left(n^{*}\right)$ is the second derivative on the top of the Gibbs free energy barrier. The attachment rate can be calculated from the mean square deviation of the cluster size at the top of the free energy barrier by

$$
f_{n^{*}}=\frac{1}{2} \frac{\left\langle[n(t)-n(0)]^{2}\right\rangle}{t},
$$

where $n(t)$ is the cluster size at time $t$. The mean square deviation of the cluster size can be determined from EDMD simulations starting from configurations at the top of the free energy barriers. Using the results for the attachment rates and 
TABLE I. Nucleation rates $I \sigma^{5} / 6 D_{l}$ for the nucleation of the plastic crystal phase in systems of hard dumbbells with elongation $L^{*}$, at pressure $P \sigma^{3} / k_{B} T$, and supersaturation $\beta|\Delta \mu|$. Here, $\rho_{s} d^{3}$ is the number density of dumbbells in the solid phase, $\beta \Delta G\left(n^{*}\right)$ is the barrier height, and $\left|\beta \Delta G^{\prime \prime}\left(n^{*}\right)\right|$ is the second derivative of the Gibbs free energy at the critical nucleus size $n^{*}$, i.e., the number of dumbbells in the critical cluster. Here, $f_{n^{*}} / 6 D_{l}$ is the attachment rate in units of the long-time diffusion coefficient $D_{l}$.

\begin{tabular}{lcccccccccc}
\hline \hline$L^{*}$ & $P \sigma^{3} / k_{B} T$ & $\beta|\Delta \mu|$ & $\rho_{s} d^{3 \mathrm{a}}$ & $n^{*}$ & $\beta \Delta G\left(n^{*}\right)$ & $\left|\beta \Delta G^{\prime \prime}\left(n^{*}\right)\right|$ & $f_{n^{*}} / 6 D_{l}$ & $D_{l} \tau / \sigma^{2}$ & $I \sigma^{5} / 6 D_{l}(\mathrm{US})$ & $I \sigma^{5} / 6 D_{l}(\mathrm{MD})$ \\
\hline 0 & 15 & 0.34 & 1.107 & 300 & $42.9 \pm 0.3$ & $5.1 \times 10^{-4}$ & 4550 & 0.012 & $9.6 \times 10^{-18 \pm 1}$ \\
0.15 & 13.8 & 0.34 & 1.104 & 265 & $44.5 \pm 1.1$ & $6.0 \times 10^{-4}$ & 3700 & 0.01 & $1.4 \times 10^{-18 \pm 1}$ \\
0.3 & 21 & 0.34 & 1.163 & 220 & $45.2 \pm 2$ & $1.0 \times 10^{-3}$ & 7464 & 0.0023 & $1.7 \times 10^{-18 \pm 1}$ & $\ldots$ \\
0 & 17 & 0.54 & 1.136 & 102 & $19.6 \pm 0.3$ & $1.2 \times 10^{-3}$ & 3980 & 0.0078 & $1.7 \times 10^{-7 \pm 1}$ & $1.6 \times 10^{-7 \mathrm{~b}}$ \\
0.15 & 16 & 0.54 & 1.131 & 70 & $18.0 \pm 0.7$ & $9.7 \times 10^{-4}$ & 3779 & 0.006 & $6.1 \times 10^{-7 \pm 1}$ & $3.5 \times 10^{-7 \pm 1}$ \\
0.2 & 17.5 & 0.54 & 1.143 & 65 & $15.8 \pm 0.5$ & $2.0 \times 10^{-3}$ & 2682 & 0.003 & $5.5 \times 10^{-6 \pm 1}$ & $4.4 \times 10^{-6 \pm 1}$ \\
\hline \hline
\end{tabular}

${ }^{\mathrm{a}} d^{3}=\sigma^{3}\left(1+3 / 2 L^{*}-1 / 2 L^{* 3}\right)($ Ref. 28$)$.

${ }^{\mathrm{b}}$ Extrapolated from Ref. 23.

the nucleation barriers obtained from umbrella sampling MC simulations, we can determine the nucleation rates, which we compare with those obtained directly from spontaneous nucleation events in EDMD simulations. We observed a large variance in the attachment rates calculated for different nuclei. We used ten independent configurations on the top of the barrier and followed ten trajectories for each of them to determine the attachment rates. Taking into account the statistical errors in the free energy barriers and attachment rates, we estimate that the error in the resulting nucleation rates is one order of magnitude. In order to exclude the effect of dynamics, we compare the nucleation rates for the plastic crystal phase in long-time diffusion times, i.e., $\tau_{L}=\sigma^{2} / 6 D_{l}$ with $D_{l}$ the long-time diffusion coefficient. We calculate $D_{l}$ by measuring the mean square displacement at supersaturation $\beta|\Delta \mu|$ $=0.34$ and 0.54 as shown in Table I for various aspect ratios. We clearly observe that the dynamics become slower for increasing aspect ratio $L^{*}$, resulting in long-time diffusion coefficients $D_{l} \tau / \sigma^{2}=0.012,0.01,0.0023$ for $L^{*}=0,0.15$ and 0.3 at $\beta|\Delta \mu|=0.34$ with $\tau=\sigma \sqrt{m / k_{B} T}$. At higher supersaturation $\beta|\Delta \mu|=0.54$, we find even smaller values for $D_{l}$, i.e., $D_{l} \tau / \sigma^{2}=0.0078,0.006,0.003$ for $L^{*}=0,0.15$ and 0.2 , respectively.

The resulting nucleation rates in units of the long-time diffusion coefficient are shown in Table I. We wish to make a few remarks here. First, the nucleation rates obtained from spontaneous nucleation events observed in EDMD simulations agree well with the ones obtained from umbrella sampling MC simulations within error bars of one order of magnitude, which means that the nucleation results obtained from the umbrella sampling MC simulations are reliable. Second, we clearly observe that the nucleation rates for the different aspect ratios ranging from $L^{*}=0$ to 0.3 are remarkably similar as the differences are within the errorbars for both supersaturations.

Finally, we made an attempt to study spontaneous nucleation of dumbbells with $L^{*}=0.3$ at supersaturation $\beta|\Delta \mu|=0.54$ using event-driven MD simulations. As already mentioned above, the plastic crystal phase for dumbbells with $L^{*}=0.3$ becomes metastable with respect to the aligned CP1 phase for $P^{*}=P \sigma^{3} / k_{B} T>30$, i.e., $\beta|\Delta \mu|$ $>0.47$. Hence, we would expect to find the nucleation of the CP1 phase here. However, we find that the nucleation is severely hampered due to slow dynamics, which can be appreciated from Fig. 2, where we plot the mean square displacement for $\beta|\Delta \mu|=0.47$. The resulting long-time diffusion coefficient $D_{l}=1.72 \times 10^{-4} \sigma^{2} / \tau$ is at least one order of magnitude smaller than the long-time diffusion coefficients at $\beta|\Delta \mu|=0.54$, where we observed spontaneous nucleation for $L^{*}=0,0.5$, and 0.2 .

In umbrella sampling MC simulations, we can "fix" the simulations at the top of the nucleation barrier which allows us to study the properties of the critical nuclei. We investigate the effect of the particle anisotropy on the structure of the critical nuclei using the order parameters $\bar{q}_{4}$ and $\bar{w}_{4}$ as defined above. At supersaturation $\beta|\Delta \mu|=0.34$, the size of the critical nuclei is $n \simeq 250$ which is sufficiently large to determine the crystal structure of the nuclei. For each dumbbell, we calculate the averaged local bond order parameter $\bar{q}_{4}$ and $\bar{w}_{4}$, provided the particle has $N_{b}(i) \geq 10$ neighbors. The distribution of particles in the critical nuclei is presented as scatter plots in the $\bar{q}_{4}-\bar{w}_{4}$ plane along with those for pure fcc and hcp plastic crystal phases of dumbbells with $L^{*}=0,0.15$, and 0.3 , at corresponding pressures. From Fig. 3, we clearly observe that the critical nuclei for $L^{*}=0$ and 0.15 contains predominantly fcc-like rather than hcp-like particles. In order to distinguish the fcc-like and hcp-like particles more quantitatively, we divide the $\bar{q}_{4}-\bar{w}_{4}$ plane by a straight line

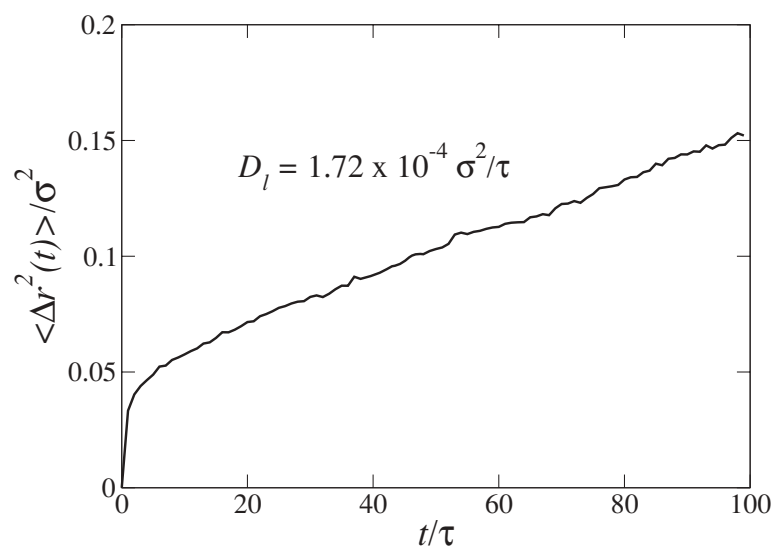

FIG. 2. Mean square displacement $\left\langle\Delta r^{2}(t)\right\rangle$ as a function of time $t / \tau$ in a fluid of hard dumbbells with $L^{*}=0.3$ at $P^{*}=30$ (for $\beta|\Delta \mu|=0.47$ ). 

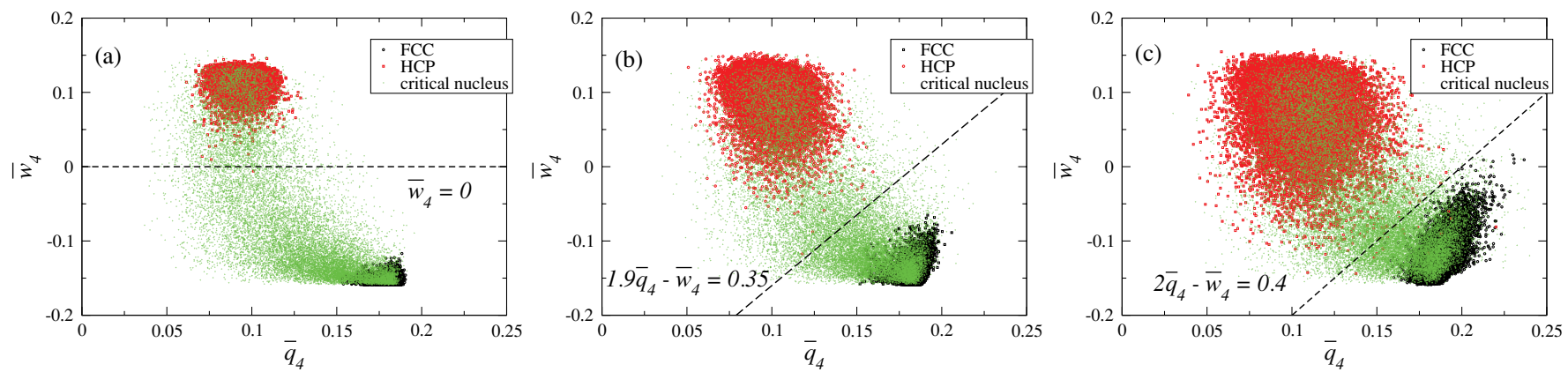

FIG. 3. Distribution of particles in the critical nuclei for the plastic crystal nucleation in hard dumbbell systems with $L^{*}=0$ (a), 0.15 (b), and 0.3 (c) as obtained from umbrella sampling MC simulations at a supersaturation $\beta|\Delta \mu|=0.34$ in the $\bar{q}_{4}-\bar{w}_{4}$ plane compared with those for pure fcc and hep plastic crystal phases with corresponding pressures. The dashed lines are used to distinguish the fcc-like and hcp-like particles, and the formulas are next to them.

in such a way that the particle distributions for the pure fcc and hcp plastic crystal phases are maximally separated. We plot the criteria to distinguish fcc-like and hcp-like particles as dashed straight lines in Fig. 3 with the corresponding formula. We note, however, that the criteria seem to be arbitrarily chosen, but the identification of fcc-like and hcp-like particles for typical nuclei seems to be less sensitive on the precise details of these criteria. Typical snapshots of the critical nuclei for $L^{*}=0,0.15$, and 0.3 are shown in Fig. 4, where the color coding denotes the identity (fcc-like, hcplike, or undetermined) of the particle using these criteria. As we did not calculate the averaged local bond order parameter $\bar{q}_{4}$ and $\bar{w}_{4}$ for particles with $N_{b}(i)<10$ neighbors, the identity of these particles remains undetermined. We clearly observe that the critical nuclei for $L^{*}=0,0.15$ contain mainly fcc-like particles. The particle distributions become broader for the pure fcc and hcp plastic crystal phases upon increasing $L^{*}$ and consequently it becomes more difficult to distinguish fcc-like and hcp-like particles. However, the fraction of hcp-like particles seems to increase with increasing particle elongation. This agrees with the results from free energy calculations of hard dumbbell systems, where it has been shown that the hcp plastic crystal phase is more stable than the one with the fcc structure at $L^{*} \geq 0.15 .{ }^{13}$ It is worth noting here that recent nucleation studies of hard spheres showed that the critical nuclei contain approximately $80 \%$ fcc-like particles. ${ }^{23}$ As the free energy difference per particle between bulk fcc and hcp phases is only about $0.001 k_{B} T$ at melting, the predominance for fcc-like particles is attributed to surface effects.

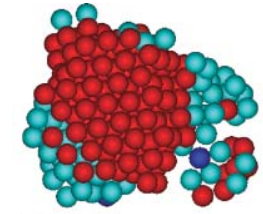

(a)

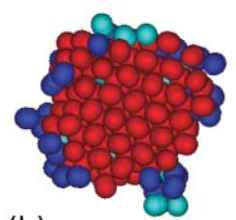

(b)

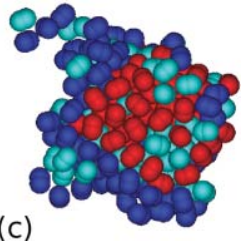

(c)

\section{B. Nucleation of the aperiodic crystal phase}

For more elongated dumbbells, i.e., $L^{*}>0.88$, the orientationally disordered aperiodic crystal phase becomes stable, ${ }^{10-13}$ in which the individual spheres of the dumbbells are on a random hcp lattice, whereas the orientations of the dumbbells are random. In this section, we investigate the nucleation of the aperiodic crystal phase of hard dumbbells with different aspect ratios. We perform Monte Carlo simulations using the umbrella sampling technique to determine the Gibbs free energy as a function of cluster size for hard dumbbells with $L^{*}=1.0$ and supersaturation $P^{*}=16$ and 17. The order parameter that is employed here in the umbrella sampling technique is equal to the number of spheres $n$ (and thus not the number of dumbbells) in the largest crystalline cluster in the system. Thus, we check for each individual sphere whether or not it belongs to the largest crystalline cluster and as a consequence, the whole dumbbell can be part of the largest cluster or only one sphere of the dumbbell can belong to the cluster, or the whole dumbbell is regarded to be fluidlike. Consequently, it is convenient to introduce a bulk chemical potential per sphere, which equals 0.5 times the bulk chemical potential per dumbbell $\mu^{\mathrm{sph}}=\mu / 2$. We compare the results with those for hard spheres at the same pressure in Fig. 5. Since the bulk pressure for the solid-fluid transition of hard dumbbells with $L^{*}=1$ is remarkably close to that

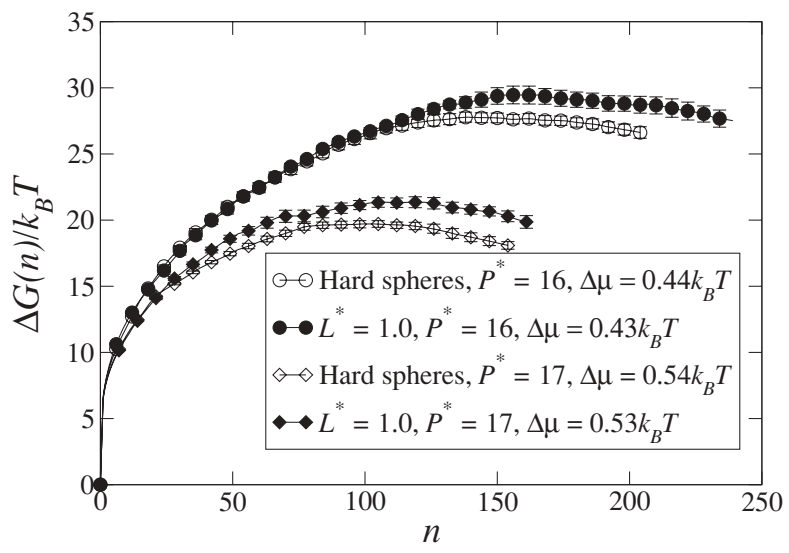

FIG. 5. Gibbs free energy $\Delta G(n)$ as a function of number of spheres $n$ in the largest cluster for the nucleation of the (aperiodic) crystal phase of hard dumbbells and of hard spheres. 


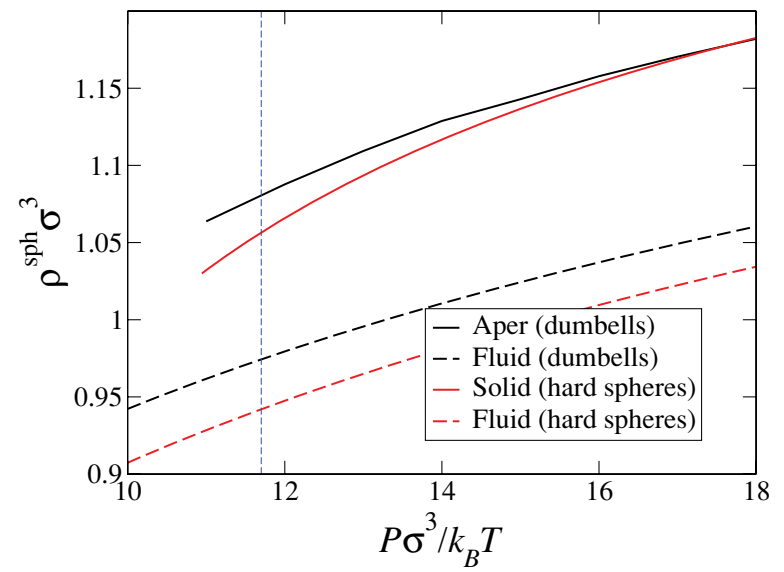

FIG. 6. Equation of state (EOS, i.e., $\beta P \sigma^{3}$ vs the number density of spheres $\rho^{\mathrm{sph}} \sigma^{3}$ for a system of hard spheres and hard dumbbells with $L^{*}=1.0$. For the fluid and solid phases of hard spheres, the Carnahan-Starling (Ref. 30) and Speedy (Ref. 31) EOS are plotted. The EOS of hard dumbbells for the fluid phase is obtained from Ref. 32. The dashed vertical line denotes the bulk coexistence pressure of hard dumbbells with $L^{*}=1.0$.

of hard spheres $\beta P_{\text {coex }} \sigma^{3}=11.8,{ }^{10-13}$ one might naively expect that the nucleation barriers should be compared at the same dimensionless pressure. However, we observe that at the same pressure, the nucleation barrier for the aperiodic crystal phase of hard dumbbells is slightly higher than that of hard spheres. CNT predicts that the barrier height is given by $\Delta G^{*}=(16 \pi / 3) \gamma^{3} /\left(\rho_{s}^{\mathrm{sph}}\left|\Delta \mu^{\mathrm{sph}}\right|\right)^{2}$, and hence a difference in barrier height should be due to a difference in the interfacial tension $\gamma$, the density of spheres in the solid phase $\rho_{s}^{\text {sph }}$, or in $\left|\Delta \mu^{\mathrm{sph}}\right|$. As the reduced density of spheres $\rho_{s}^{\mathrm{sph}} \sigma^{3}$ in the aperiodic crystal phase is very close to that of a solid phase of hard spheres at $P^{*}=16$ and 17, and the interfacial tensions $\beta \gamma \sigma^{2}$ are also expected to be very similar, the difference in barrier height can only be caused by a difference in $\left|\Delta \mu^{\mathrm{sph}}\right|$. We, therefore, calculated more accurately the bulk chemical potential difference per sphere between the solid and the fluid phase using

$$
\left|\Delta \mu^{\mathrm{sph}}\right|=\int_{P_{\text {coex }}}^{P}\left(\frac{1}{\rho_{l}^{\mathrm{sph}}}-\frac{1}{\rho_{s}^{\mathrm{sph}}}\right) \mathrm{d} P,
$$

where $\rho_{l}^{\text {sph }}$ and $\rho_{s}^{\text {sph }}$ are the density of spheres in the liquid and solid phases. In Fig. 6, we plot the equation of state for the fluid and solid phases of hard spheres from Refs. 30 and 31 along with the equation of state for the fluid phase of hard dumbbells for $L^{*}=1$ from Ref. 32. In addition, we determined the equation of state for the solid phase using EDMD simulations. Using these results and Eq. (10), we indeed find that the supersaturation $\beta \mid \Delta \mu^{\mathrm{sph}}$ per sphere is $\sim 2.3 \%$ smaller for hard dumbbells than for hard spheres, resulting in an increase in barrier height of $\sim 5 \%$, which perfectly matches our results. We conclude that the difference in the height of the nucleation barrier between the aperiodic crystal phase of dumbbells with $L^{*}=1.0$ and the hard-sphere crystal is mostly due to the difference in $\left|\Delta \mu^{\mathrm{sph}}\right|$.

Moreover, we also performed EDMD simulations for the spontaneous nucleation of the aperiodic crystal phase of

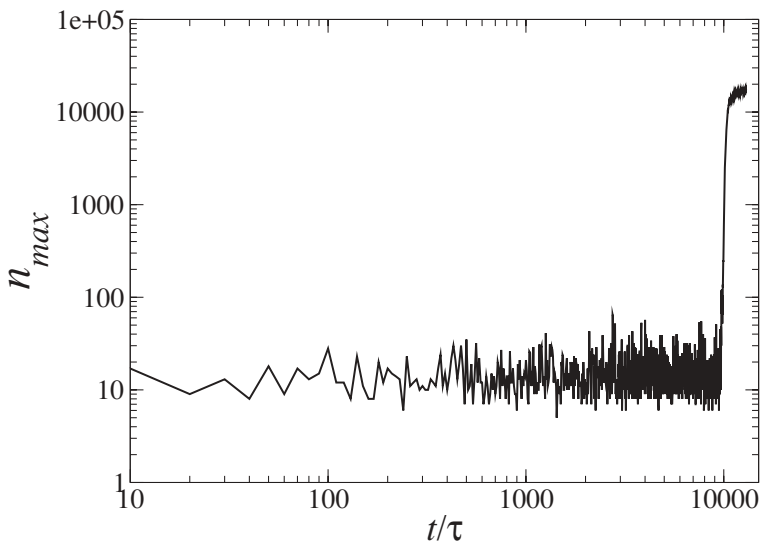

FIG. 7. Number of spheres in the largest cluster $n_{\max }$ as a function of time $t / \tau$ for a typical trajectory obtained from EDMD simulations for the aperiodic crystal nucleation of hard dumbbells with $L^{*}=1.0, N=16000$, and $P^{*}=17$

dumbbells at $P^{*}=17$ in system of $N=16000$ hard dumbbells. The number of spheres in the biggest cluster as a function of time from a typical MD simulation is shown in Fig. 7. We find that the size of critical nuclei in spontaneous nucleation is around 100 spheres which agrees well with the result obtained from umbrella sampling MC simulations shown in Fig. 5. The nucleation rate obtained from spontaneous nucleation events observed in MD simulations is $I \sigma^{5} / 6 D_{l}=7.3$ $\times 10^{-8 \pm 1}$ which agrees very well with the rate obtained from umbrella sampling MC simulations, $I \sigma^{5} / 6 D_{l}=2.8$ $\times 10^{-8 \pm 1}$, within the error bars of one order of magnitude.

Furthermore, we study the effect of aspect ratio on the nucleation of the aperiodic crystal phase and the free energy barriers for hard dumbbells with aspect ratios $L^{*}=0.95,0.97$, and 1.0 at supersaturation $\beta\left|\Delta \mu^{\mathrm{sph}}\right|=0.43$. We plot $\Delta G(n)$ as a function of cluster size $n$, i.e., the number of spheres in the cluster, in Fig. 8. We observe that at the same supersaturation, the barrier height decreases upon decreasing the elongation of the dumbbells. According to classical nucleation theory, $\Delta G^{*} \propto \gamma^{3} /\left(\rho_{s}^{\mathrm{sph}}\left|\Delta \mu^{\mathrm{sph}}\right|\right)^{2}$, where $\Delta \mu^{\mathrm{sph}}$ is the supersaturation per sphere with $\rho_{s}^{\text {sph }}$ the bulk density of

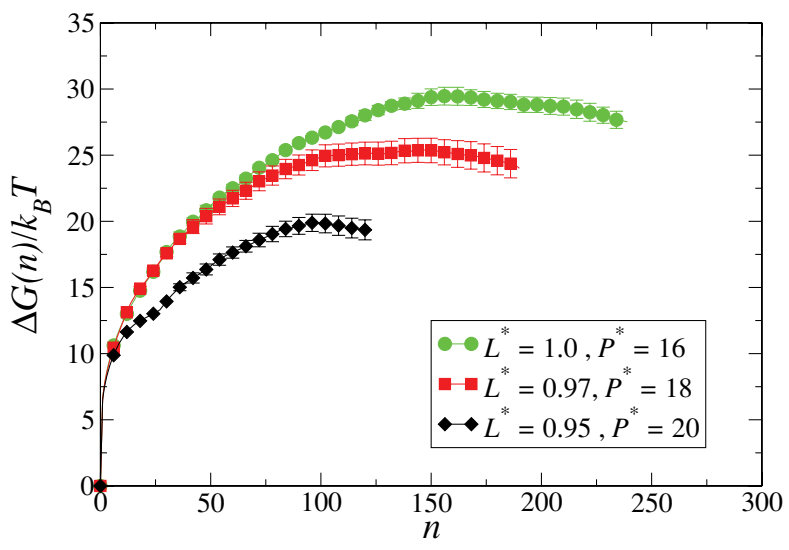

FIG. 8. Gibbs free energy $\Delta G(n)$ as a function of the number of spheres $n$ in the largest crystalline cluster for the aperiodic crystal nucleation of hard dumbbells with $L^{*}=0.95,0.97$, and 1 at supersaturation $\beta\left|\Delta \mu^{\mathrm{sph}}\right|=0.43$. 
TABLE II. Nucleation rates $I \sigma^{5} / 6 D_{l}$ for the nucleation of the aperiodic crystal phase in systems of hard dumbbells with elongation $L^{*}$, at pressure $P \sigma^{3} / k_{B} T$, and supersaturation per sphere $\beta\left|\Delta \mu^{\mathrm{sph}}\right|$. Here, $\rho_{s}^{\mathrm{sph}} \sigma^{3}$ is the number density of spheres in the solid phase, $\beta \Delta G\left(n^{*}\right)$ is the barrier height, and $\left|\beta \Delta G^{\prime \prime}\left(n^{*}\right)\right|$ is the second derivative of the Gibbs free energy at the critical nucleus size $n^{*}$, i.e., the number of spheres in the critical cluster. Here, $f_{n^{*}} / 6 D_{l}$ is the attachment rate in units of the long-time diffusion coefficient $D_{l}$.

\begin{tabular}{lcccccccccc}
\hline \hline$L^{*}$ & $P \sigma^{3} / k_{B} T$ & $\beta\left|\Delta \mu^{s p h}\right|$ & $\rho_{s}^{s p h} \sigma^{3}$ & $n^{*}$ & $\beta \Delta G\left(n^{*}\right)$ & $\left|\beta \Delta G^{\prime \prime}\left(n^{*}\right)\right|$ & $f_{n^{*}} / 6 D_{l}$ & $D_{l} \tau / \sigma^{2}$ & $I \sigma^{5} / 6 D_{l}(\mathrm{US})$ & $I \sigma^{5} / 6 D_{l}(\mathrm{MD})$ \\
\hline 1 & 17 & 0.53 & 1.170 & 115 & $21.4 \pm 0.4$ & $1.2 \times 10^{-3}$ & 2813 & 0.0026 & $2.0 \times 10^{-8 \pm 1}$ & $7.3 \times 10^{-8 \pm 1}$ \\
1 & 16 & 0.43 & 1.158 & 170 & $29.5 \pm 0.6$ & $9.4 \times 10^{-4}$ & 5556 & 0.0036 & $1.1 \times 10^{-11 \pm 1}$ & $\ldots$ \\
0.97 & 18 & 0.43 & 1.171 & 140 & $25.3 \pm 0.9$ & $8.4 \times 10^{-4}$ & 5228 & 0.0022 & $6.6 \times 10^{-10 \pm 1}$ & $\ldots$ \\
0.95 & 20 & 0.43 & 1.182 & 100 & $19.9 \pm 0.7$ & $3.0 \times 10^{-3}$ & 2273 & 0.0011 & $1.2 \times 10^{-7 \pm 1}$ & $\ldots$ \\
\hline \hline
\end{tabular}

spheres in the solid phase. As shown in Table II, $\rho_{s}^{\mathrm{sph}} \sigma^{3}$ is very similar for $L^{*}=0.95,0.97$, and 1.0 , and we argue that the interfacial tension of the aperiodic crystal decreases upon decreasing the elongation of the dumbbells. In order to calculate the nucleation rates, we perform EDMD simulations starting from configurations on the top of the free energy barriers. We plot the mean square deviation of the cluster size as a function of time in Fig. 9. We find that the attachment rate decreases significantly as the anisotropy of the dumbbells decreases. The resulting nucleation rates in units of the long time diffusion coefficient are shown in Fig. 10. We clearly observe that at fixed supersaturation the nucleation rate increases with decreasing dumbbell elongation. However, in the phase diagram of hard dumbbells, ${ }^{10-13}$ the pressure range where the aperiodic crystal phase is thermodynamically stable shrinks significantly when the aspect ratio decreases. As a result, it is not possible to increase the supersaturation further for shorter dumbbells, although the nucleation rates are already much higher for shorter ones than for longer ones at the same supersaturation.

Additionally, we also study the structure of the critical nuclei by calculating the averaged local bond order parameter $\bar{q}_{4}$ and $\bar{w}_{4}$, provided the sphere has $N_{b}(i) \geq 10$ neighbors. The distribution of spheres in the critical nuclei is presented as scatter plots in the $\bar{q}_{4}-\bar{w}_{4}$ plane in Fig. 11 for $L^{*}=1.0$. We observe only a few spheres with $\bar{w}_{4}>0$ and $\bar{q}_{4}$ $<0.1$, as most of the spheres are in the area of $\bar{w}_{4}<0$ and $\bar{q}_{4}>0.1$, which is very similar to the scatter plots for hard

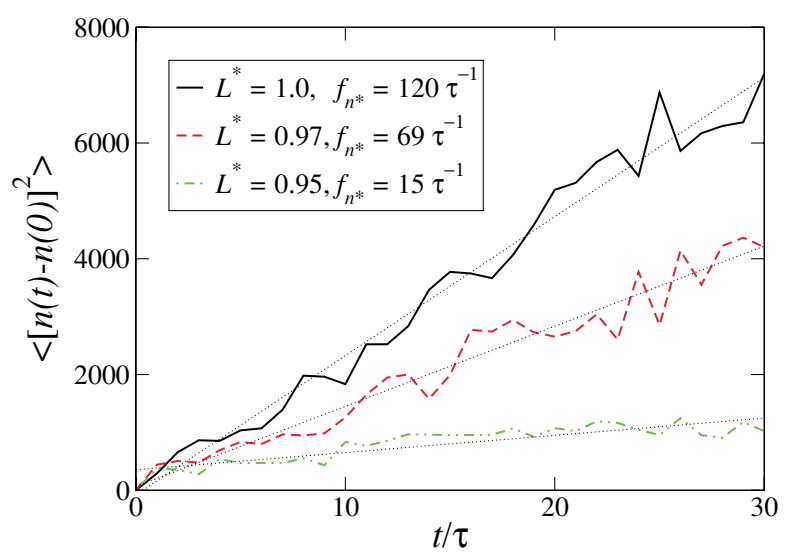

FIG. 9. Mean square deviation of the cluster size $\left\langle[n(t)-n(0)]^{2}\right\rangle$ as a function of time $t / \tau$ for hard dumbbells with $L^{*}=0.95,0.97$, and 1.0 at supersaturation $\beta\left|\Delta \mu^{\mathrm{sph}}\right|=0.43$. The resulting attachment rates $f_{n^{*}}$ are listed in units of $\tau^{-1}$. spheres shown in Fig. 3(a). Consequently, the critical nucleus of the aperiodic crystal phase of hard dumbbells contains also more fcc-like than hcp-like particles, similar to the critical nuclei observed in hard-sphere nucleation. ${ }^{23}$ A typical configuration of a critical nucleus is shown in the inset of Fig. 11, where the spheres are considered to be fcc-like if $\bar{w}_{4}<0$.

\section{Slow dynamics of hard dumbbells}

The phase diagram of hard dumbbells shows a stable aligned CP1 crystal phase at infinite pressure for all aspect ratios of the dumbbells and a fluid-CP1 coexistence region for $0.4 \leq L^{*} \leq 0.8 .^{10-13}$ The surface tension for the fluidCP1 interface of hard dumbbells with $L^{*}=0.4$ is $\beta \gamma \sigma^{2}$ $\simeq 1.8 .^{28}$ The height of free energy barrier is given by $\Delta G^{*}$ $=16 \pi \gamma^{3} / 3\left(\rho_{s}|\Delta \mu|\right)^{2}$ in CNT. If we assume that the interfacial tension does not change significantly with increasing pressure, we can estimate the free energy barrier height as a function of pressure by integrating the Gibbs-Duhem equation to obtain $|\Delta \mu|$. The barrier height $\Delta G^{*}$ and the packing fraction $\eta$ for the fluid phase are shown in Fig. 12 as a function of the pressure $P^{*}$. We find that the barrier height $\Delta G^{*}$ is extremely high, and only becomes less than $50 k_{B} T$ for $P^{*}>45$, corresponding to a packing fraction of the fluid phase $\eta>0.67$. However, if the interfacial tension increases with increasing pressure as shown in Ref. 33, the "actual" height of free energy barrier can become even higher. As a

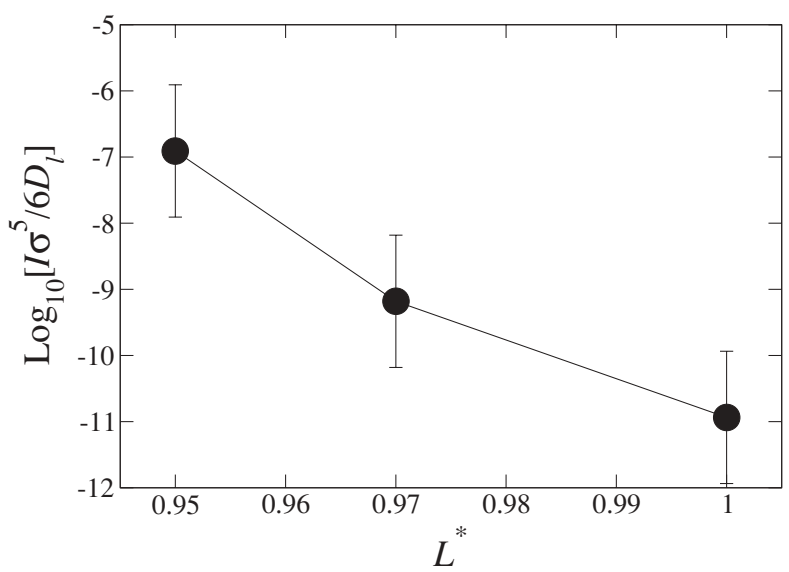

FIG. 10. Nucleation rate $I \sigma^{5} / 6 D_{l}$ for the aperiodic crystal phase as a function of the aspect ratio $L^{*}$ of hard dumbbells at supersaturation $\beta\left|\Delta \mu^{\mathrm{sph}}\right|$ $=0.43$. 


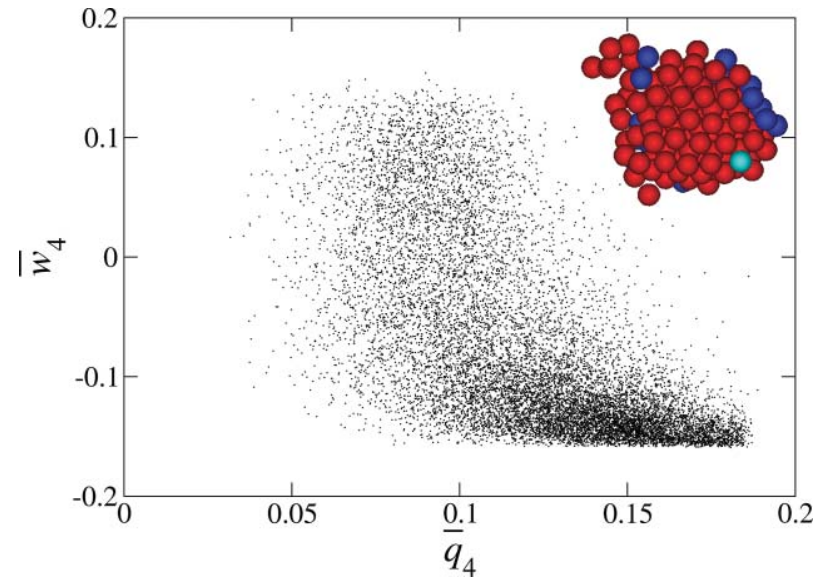

FIG. 11. Distribution of the spheres in the critical nuclei as obtained from umbrella sampling MC simulations in $\bar{q}_{4}-\bar{w}_{4}$ plane in systems of hard dumbbells with $L^{*}=1.0$ at supersaturation $\beta\left|\Delta \mu^{\mathrm{sph}}\right|=0.43$. Inset: Typical configuration of a critical nucleus. Red denotes fcc-like spheres and blue denotes hcp-like spheres, while the light blue are the undetermined ones.

consequence, nucleation of the $\mathrm{CP} 1$ crystal phase is an extremely rare event.

Additionally, we calculate mean square displacements $\left\langle\Delta r^{2}(t)\right\rangle$ and the second-order orientational correlator $L_{2}(t)=\left\langle P_{2}[\cos (\theta(t))]\right\rangle$ for a metastable fluid of hard dumbbells with $L^{*}=0.4,0.5$, and 0.8 at supersaturation $\beta|\Delta \mu|=$ 1.0 as shown in Fig. 13. We find that at a supersaturation $\beta|\Delta \mu|=1.0$, where the barrier height is still very high, $\Delta G^{*} / k_{B} T \sim 170$ for $L^{*}=0.4$, the long-time diffusion coefficients $D_{l} \simeq 10^{-4} \sigma^{2} / \tau$ obtained from $\left\langle\Delta r^{2}(t)\right\rangle$ is extremely small (see Table III), whereas $L_{2}(t)$ exhibits slow relaxation. Our findings are consistent with predictions obtained from mode-coupling theory for a liquid-glass transition, in which the structural arrest is due to steric hindrance for both translational and reorientational motion. ${ }^{34-38}$ Moreover, mode-coupling theory predicts that the steric hindrance for reorientations becomes stronger with increasing elongation, which is consistent with our results for $L_{2}(t)$ in Fig. 13. ${ }^{34-38}$ Increasing the supersaturation will lower $\Delta G^{*}$,

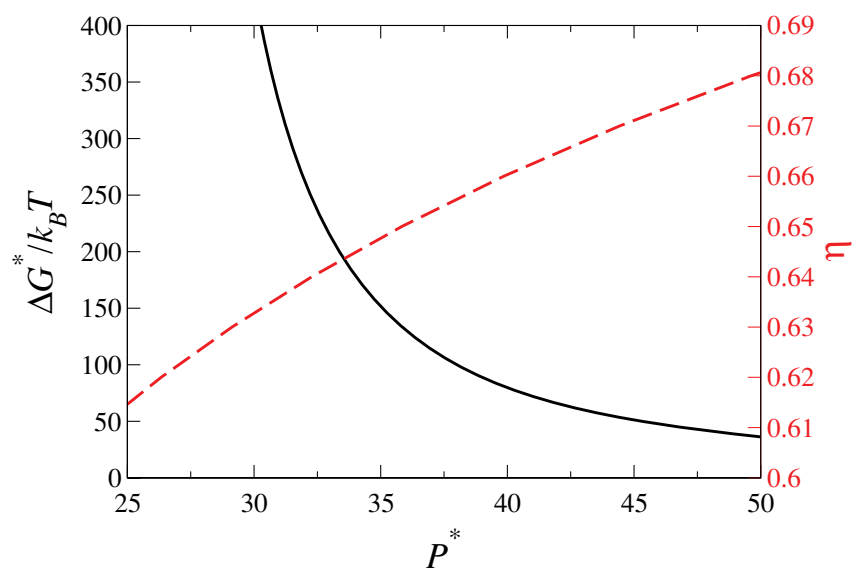

FIG. 12. Estimated height of the Gibbs free energy barrier $\Delta G^{*} / k_{B} T$ obtained from classical nucleation theory (solid line) and the packing fraction $\eta$ in the supersaturated fluid phase (Ref. 32) (dashed line) as a function of pressure $P^{*}$ for the nucleation of the CP1 phase of hard dumbbells with $L^{*}=0.4$.

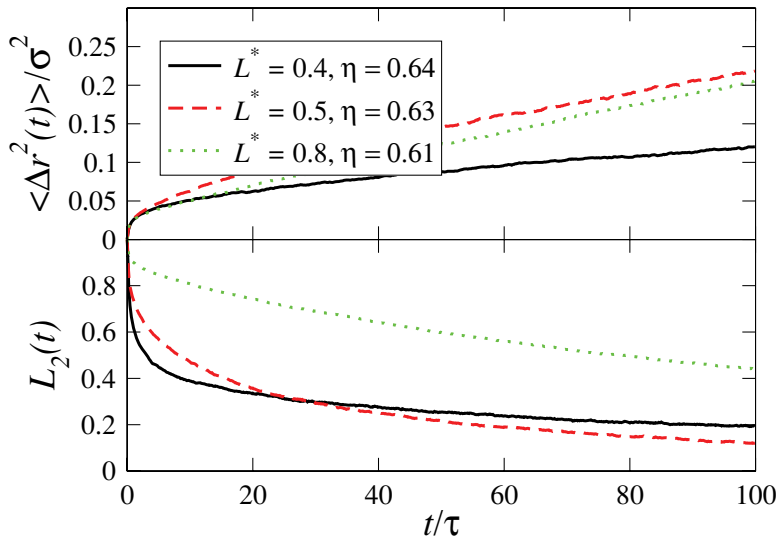

FIG. 13. Mean square displacements $\left\langle\Delta r^{2}(t)\right\rangle$ and $L_{2}(t)=\left\langle P_{2}[\cos (\theta(t))]\right\rangle$ as a function of time for a fluid of hard dumbbells with an aspect ratio $L^{*}$ $=0.4,0.5$, and 0.8 at supersaturation $\beta|\Delta \mu|=1.0$.

but $D_{l}$ will decrease as well, while at lower supersaturation the barrier height will only increase. As a result, the nucleation of CP1 phase of hard dumbbells is severely hindered by a high free energy barrier at low supersaturations and slow dynamics at high supersaturations, which explains why the CP1 phase of colloidal hard dumbbells has never been observed in experiments ${ }^{18}$ or in direct simulations. It is worth noting that the phase diagram might also display (meta)stable CP2 and CP3 close-packed crystal structures, ${ }^{10}$ which only differ in the way the hexagonally packed dumbbell layers are stacked. As the free energy difference for the three close-packed structures is extremely small, we expect the surface tensions and the nucleation barrier height to be very similar. Hence, we expect that also the nucleation of the $\mathrm{CP} 2$ and $\mathrm{CP} 3$ phases are hindered by either a high free energy barrier or slow dynamics.

\section{CONCLUSIONS}

In conclusion, we investigated the homogeneous nucleation of the plastic crystal, aperiodic crystal, and CP1 crystal phase of hard dumbbells using computer simulations. Hard dumbbells serve as a model system for colloidal dumbbells for which the self-assembly is mainly determined by excluded volume interactions. For charged colloidal dumbbells or diatomic molecules, screened Coulombic interactions and Van der Waals interactions may significantly change the kinetic pathways for nucleation. For instance, crystal nucleation of hard rods proceeds via multilayered crystalline nuclei, whereas attractive depletion interactions between the

TABLE III. Long-time diffusion coefficients $D_{l}$ in units of $\sigma^{2} / \tau$ with $\tau=$ $\sigma \sqrt{m / k_{B} T}$ for hard dumbbells with elongation $L^{*}$ at pressure $P^{*}$, packing fraction $\eta$, and supersaturation $\beta|\Delta \mu|=1.0$.

\begin{tabular}{cccc}
\hline \hline$L^{*}$ & $P^{*}$ & $\eta$ & $D_{l} \tau / \sigma^{2}$ \\
\hline 0.4 & 34.5 & 0.64 & $1.02 \times 10^{-4}$ \\
0.5 & 31.2 & 0.63 & $2.47 \times 10^{-4}$ \\
0.8 & 24.8 & 0.61 & $2.78 \times 10^{-4}$ \\
\hline \hline
\end{tabular}


rods in a polymer solutions favor the nucleation of singlelayered nuclei. ${ }^{20,39}$ For the nucleation of the plastic crystal phase of hard dumbbells, we found that at low supersaturations the free energy barriers increase slightly with increasing dumbbell anisotropy, which can be explained by a small increase in surface tension for more anisotropic dumbbells. ${ }^{28}$ When the supersaturation increases, the barrier height decreases with increasing dumbbell aspect ratio, which can only be explained by a different pressure dependence of the interfacial tension for hard dumbbells with different aspect ratios. Although the nucleation rate for the plastic crystal phase does not vary much with aspect ratio, the dynamics do decrease significantly. We also carried out EDMD simulations and compared the nucleation rates obtained from spontaneous nucleation events with those obtained from the umbrella sampling Monte Carlo simulations and found good agreement within the error bars of one order of magnitude. Additionally, we investigated the structure of the critical nuclei of the plastic crystal phase of hard dumbbells with various aspect ratios. We found that the nuclei of the plastic crystal tend to include more fcc-like particles rather than hcp-like ones, which is similar to the critical nuclei of hard spheres. ${ }^{23}$ However, the amount of hcp-like particles increases with increasing dumbbell aspect ratio, which agrees with the free energy calculations ${ }^{13}$ where it has been shown that the hep structure is more stable than fcc structure for $L^{*} \geq 0.15$.

Moreover, we also studied the nucleation of the aperiodic crystal phase of hard dumbbells, and our results showed that at the same pressure, the nucleation barrier of the aperiodic crystal phase of hard dumbbells with $L^{*}=1.0$ is slightly higher than that of hard spheres which is mostly due to a small difference in supersaturation $\beta\left|\Delta \mu^{\mathrm{sph}}\right|$. We also performed EDMD simulations for the spontaneous nucleation of the aperiodic crystal from hard dumbbell fluid phase, and we found that the nucleation rate obtained from spontaneous nucleation agrees very well with the one obtained from umbrella sampling MC simulations. Furthermore, we studied the effect of aspect ratio on the nucleation of the aperiodic crystal phase and found that at the same supersaturation, the nucleation rate in units of long-time diffusion coefficients increases for shorter hard dumbbells. However, when the aspect ratio of dumbbells decreases, the pressure range where the aperiodic crystal phase is stable becomes smaller. Additionally, we also found that the structure of the critical nuclei of the aperiodic crystal phase formed by hard dumbbells with $L^{*}=1.0$ is very similar to that of hard spheres which tend to have more fcclike particles rather than hcp-like ones.

We estimated the height of the free energy barrier for the nucleation of the CP1 crystal phase of hard dumbbells according to classical nucleation theory, which turns out to be extremely high in the normal pressure range due to a high interfacial tension. Furthermore, we calculated the long-time diffusion coefficients for hard dumbbells at a moderate supersaturation, i.e., $\beta|\Delta \mu|=1.0$, which appears to be very small. As a result, we conclude that the high free energy barrier as well as the slow dynamics suppress significantly the nucleation of $\mathrm{CP} 1$ phase.

\section{ACKNOWLEDGMENTS}

We thank Matthieu Marechal for offering the equation of state for the crystal structures of hard dumbbell particles and fruitful discussions. Financial support of a vici grant from the Nederlandse Organisatie voor Wetenschappelijk Onderzoek (NWO) is acknowledged.

${ }^{1}$ S. C. Glotzer and M. J. Solomon, Nature Mater. 6, 557 (2007).

${ }^{2}$ D. Zerrouki, J. Baudry, D. Pine, P. Chaikin, and J. Bibette, Nature (London) 455, 380 (2008).

${ }^{3}$ L. Filion, M. Marechal, B. van Oorschot, D. Pelt, F. Smallenburg, and M. Dijkstra, Phys. Rev. Lett. 103, 188302 (2009).

${ }^{4}$ Y. Xia, B. Gates, and Z. Li, Adv. Mater. 13, 109 (2001)

${ }^{5}$ Z.-Y. Li, J. Wang, and B.-Y. Gu, Phys. Rev. B 58, 3721 (1998).

${ }^{6}$ I. D. Hosein, M. Ghebrebrhan, J. D. Joannopoulos, and C. M. Liddell, Langmuir 26, 2151 (2010).

${ }^{7}$ S. J. Smithline, S. W. Rick, and A. D. J. Haymet, J. Chem. Phys. 88, 2004 (1988).

${ }^{8}$ J. D. McCoy, S. Singer, and D. Chandler, J. Chem. Phys. 87, 4853 (1987).

${ }^{9}$ S. Singer and R. Mumaugh, J. Chem. Phys. 93, 1278 (1990).

${ }^{10}$ C. Vega, E. P. A. Paras, and P. A. Monson, J. Chem. Phys. 96, 9060 (1992).

${ }^{11}$ C. Vega, E. P. A. Paras, and P. A. Monson, J. Chem. Phys. 96, 8543 (1992).

${ }^{12}$ C. Vega and P. A. Monson, J. Chem. Phys. 107, 2969 (1997).

${ }^{13}$ M. Marechal and M. Dijkstra, Phys. Rev. E 77, 061405 (2008).

${ }^{14}$ P. M. Johnson, C. M. van Kats, and A. van Blaaderen, Langmuir 21, 11510 (2005).

${ }^{15}$ E. Mock and C. Zukoski, Langmuir 23, 8760 (2007).

${ }^{16}$ S. H. Lee, S. J. Gerbode, B. S. John, A. K.Wolfgang, F. A. Escobedo, I. Cohen, and C. M. Liddell, J. Mater. Chem. 18, 4912 (2008).

${ }^{17}$ I. Hosein, B. S. John, S. H. Lee, F. A. Escobedo, and C. M. Liddell, J. Mater. Chem. 19, 344 (2009).

${ }^{18}$ A. F. Demirörs, P. M. Johnson, C. M. van Kats, A. van Blaaderen, and A. Imhof, Langmuir 26, 14466 (2010)

${ }^{19}$ T. Schilling and D. Frenkel, Phys. Rev. Lett. 92, 085505 (2004).

${ }^{20}$ R. Ni, S. Belli, R. van Roij, and M. Dijkstra, Phys. Rev. Lett. 105, 088302 (2010).

${ }^{21}$ P. J. Steinhardt, D. R. Nelson, and M. Ronchetti, Phys. Rev. B 28, 784 (1983).

${ }^{22}$ P. R. tenWolde, M. J. Ruiz-Montero, and D. Frenkel, Faraday Discuss. 104, 93 (1996).

${ }^{23}$ L. Filion, M. Hermes, R. Ni, and M. Dijkstra, J. Chem. Phys. 133, 244115 (2010).

${ }^{24}$ W. Lechner and C. Dellago, J. Chem. Phys. 128, 114707 (2008).

${ }^{25}$ S. Auer and D. Frenkel, Nature (London) 409, 1020 (2001).

${ }^{26}$ A. Donev, S. Torquato, and F. H. Stillinger, J. Comput. Phys. 202, 737 (2005).

${ }^{27}$ A. Donev, S. Torquato, and F. H. Stillinger, J. Comput. Phys. 202, 765 (2005).

${ }^{28}$ Y. Mu and X. Song, Phys. Rev. E 74, 031611 (2006).

${ }^{29}$ R. Davidchack, J. Morris, and B. Laird, J. Chem. Phys. 125, 094710 (2006).

${ }^{30}$ N. F. Carnahan and K. E. Starling, J. Chem. Phys. 51, 635 (1969).

${ }^{31}$ R. J. Speedy, J. Phys.: Condens. Matter 10, 4387 (1998).

${ }^{32}$ D. J. Tildesley and W. B. Streett, Mol. Phys. 41, 85 (1980).

${ }^{33}$ S. Auer and D. Frenkel, Nature (London) 413, 711 (2001).

${ }^{34}$ S.-H. Chong and W. Götze, Phys. Rev. E 65, 041503 (2002)

${ }^{35}$ S.-H. Chong and W. Götze, Phys. Rev. E 65, 051201 (2002).

${ }^{36}$ S.-H. Chong, A. J. Moreno, F. Sciortino, and W. Kob, Phys. Rev. Lett. 94, 215701 (2005).

${ }^{37}$ A. J. Moreno, S.-H. Chong, W. Kob, and F. Sciortino, J. Chem. Phys. 123, 204505 (2005).

${ }^{38}$ S.-H. Chong and W. Kob, Phys. Rev. Lett. 102, 025702 (2009).

${ }^{39}$ A. Patti and M. Dijkstra, Phys. Rev. Lett. 102, 128301 (2009). 